\title{
Surveillance for Progressive Intellectual and Neurological Deterioration in the Canadian Paediatric Population
}

\author{
Daniel L. Keene, Terry Sutcliffe, Pat Harman, and Danielle Grenier on behalf of \\ the Canadian Paediatric Surveillance Program
}

\begin{abstract}
Objectives: To conduct active surveillance of the Canadian paediatric population for children who have a progressive intellectual and neurological deterioration to detect the occurrence of cases of Creutzfeldt-Jakob disease or variant Creutzfeldt-Jakob disease. Case Definition: Any child who is less than or equal to 18 years of age, who had a progressive loss of already attained intellectual/ developmental abilities and development of abnormal neurological signs of greater than three months duration was eligible for inclusion. Duration: July 1999 to July 2001. Method: Enhanced active surveillance system for progressive intellectual and neurological deterioration was implemented to detect, prospectively, among the Canadian paediatric population. Each month, all paediatricians and paediatric neurologists in Canada were mailed a reporting form. All reported cases were reviewed by the principal investigator who classified the cases into one of four predetermined categories. Cases where there was evidence of neurological and intellectual regression without known cause were reviewed by a panel. Reported cases were reviewed for the possibility of classic or variant Creutzfeldt-Jakob disease. Results: Over 2200 physicians took part in this program. There was more than an $80 \%$ monthly return rate of the initial report form. Ninety-nine possible cases of progressive neurological and intellectual deterioration were reported. Sixty cases were classified as having a progressive neurological syndrome associated with intellectual deterioration. Fourteen cases were duplicates. One case of Creutzfeldt-Jacob disorder was found but no cases of the variant form of Creutzfeldt-Jacob disorder. Fifteen cases were felt not to meet the above-mentioned entry criteria.
\end{abstract}

RÉSUMÉ: Surveillance de la détérioration intellectuelle et neurologique progressive dans la population pédiatrique Canadienne. Objectifs: Le but de cette étude était d'effectuer une surveillance active de la population pédiatrique Canadienne pour identifier les enfants qui présentent une détérioration intellectuelle et neurologique progressive afin de détecter l'apparition de cas de maladie de Creutzfeldt-Jakob (MCJ) ou de la forme variante de la maladie vMCJ. Définition de cas: Les critères d'inclusion étaient les suivants: avoir 18 ans ou moins, présenter une perte progressive des capacités intellectuelles ou du niveau de développement atteint et apparition de signes neurologiques anormaux depuis plus de trois mois. Durée: De juillet 1999 à juillet 2001. Méthode: Un système de surveillance active accrue pour détecter de façon prospective la détérioration intellectuelle et neurologique progressive dans la population pédiatrique Canadienne a été mis en place. Tous les pédiatres et les neurologues pédiatriques du Canada ont reçu chaque mois un formulaire de notification des cas. L'investigateur principal a révisé tous les cas rapportés et les a classifiés en quatre catégories prédéterminées. Un groupe d'experts a révisé les cas qui présentaient des signes de régression neurologique et intellectuelle sans cause connue, pour évaluer la possibilité qu'il s'agisse de cas de MCJ classique ou de la forme variante de la maladie. Résultats: Plus de 2200 médecins ont participé à ce programme, avec un taux de plus de $80 \%$ de retour mensuel du formulaire initial. Quatre-vingt-dix-neuf cas de détérioration neurologique et intellectuelle progressive ont été rapportés. Soixante cas ont été classifiés comme étant des cas présentant un syndrome neurologique progressif accompagné de détérioration intellectuelle. Quatorze cas ont été rapportés plus d'une fois. Un cas de MCJ a été identifié et aucun cas de vMCJ n'a été identifié. Les critères d'inclusion n'étaient pas présents chez quinze cas.

Can. J. Neurol. Sci. 2004; 31: 220-224

While there are many disorders that present similarly with progressive intellectual and neurological deterioration (PIND) in childhood, individually their numbers are few and their diagnoses difficult to confirm. This has resulted in a general misconception that they are of minimal public health importance, yet this is clearly not the case. In the world of surveillance, acute flaccid paralysis is used to monitor polio eradication. Thus surveillance for PIND can be used to better monitor CreutzfeldtJakob disease (CJD) and variant Creutzfeldt-Jakob disease (vCJD). While CJD is rare, particularly in the paediatric population, the occurrence of vCJD in Canada would signal an important change in its epidemiology. Creutzfeldt-Jakob disease

From the Children's Hospital of Eastern Ontario, (DLK); CJD Surveillance System Canada, Health Canada, (TS, PH); Canadian Paediatric Society, (DG); Ottawa, Ontario, Canada.

ReCEIVEd June 10, 2003. ACCePted INFinAlform November 17, 2003. Reprint requests to: Daniel L. Keene, Division of Neurology, Department of Paediatrics, Children's Hospital of Eastern Ontario, 401 Smyth Road, Ottawa, Ontario Canada K1H 8L1. 
in the paediatric population has been linked to transmission through human derived growth hormone and human dura mater grafts. More recently in the United Kingdom (UK), the variant form has been reported in adolescents and young adults and linked to the consumption of beef. At the time of this study, Canada was a bovine spongiform encephalopathy "free country". This is no longer correct. To better define the clinical profile of this disease in Canada and to serve as a sentinel country for the UK, a broader surveillance approach was taken to confirm that CJD and vCJD had not gone unrecognized in Canada. For this reason, surveillance for all cases of PIND in childhood and adolescents was undertaken through the Canadian Paediatric Surveillance Program (CPSP), an active epidemiological surveillance tool designed specifically to look at rare paediatric conditions with high morbidity and mortality.

This paper reports on the results of this surveillance study focusing on three specific objectives:

1) To classify the different etiologies causing PIND in the Canadian paediatric population;

2) To review and investigate all reported cases of PIND in order to detect if any cases of CJD or vCJD had occurred in this population in Canada;

3) To enroll, upon identification, any cases of either CJD or vCJD in the CJD Surveillance System Canada, (Health Canada).

\section{METHOD}

\section{Case definition}

To be eligible for inclusion in this study, the child had to have been less than or equal to 18 years of age, residing in Canada, and have demonstrated progressive loss of intellectual or developmental abilities in association with abnormal neurological signs for a period of more than three months. Children with known neurological disorders secondary to head injury, acute infection of the central nervous system, prenatal hypoxic-ischemic insult, neoplasm or near drowning were excluded, as were children with autism or epileptic encephalopathies.

\section{Duration of the study}

The study was undertaken from July 1999 to June 2001.

\section{Case ascertainment}

This study was done in co-operation with the CPSP, ${ }^{1}$ a joint project of the Canadian Paediatric Society and the Centre for Infectious Disease Prevention and Control, Health Canada, first established in 1996. The CPSP utilizes a two-tier reporting methodology for ascertainment and investigation, beginning with an initial monthly report form for case ascertainment and followed by a detailed questionnaire requesting limited nonnominal data sufficient to allow for case confirmation. Prior to the start of the study, the case definition and protocol were mailed to ensure that responding physicians understood the methodology, process and criteria necessary to confirm a case. Then, on a monthly basis, the CPSP sent an initial report form listing conditions under surveillance to all practicing paediatricians, medical geneticists and paediatric neurologists in Canada. The responding physician was asked to indicate either a 'nil'report, or the number of new cases seen during the previous month that met the study case definition. A postage-paid return envelope was provided to return the form to the CPSP. Quarterly reminders were mailed to all respondents who had not replied for any given month during the study period. Upon receipt of the initial case report, the CPSP senior coordinator forwarded the information to the study's senior author and mailed a more detailed clinical questionnaire to the reporting physician. The questionnaire asked for a brief synopsis of the clinical history, physical findings, investigations and diagnosis (if any). Only non-nominal data such as the patient's date of birth and sex were collected to identify duplicate reports. Reminders were sent to all reporting physicians who had not returned the detailed questionnaire. The senior author contacted reporting physicians directly by telephone to obtain missing information as needed.

Cases were reviewed and classified on a regular basis with the help of a panel of medical experts consisting of four paediatric neurologists, a medical geneticist, and a paediatric neuropathologist. In cases where the diagnosis was in doubt or clinical history was unclear, the reporting physician was invited to participate in a teleconference with the expert panel in an attempt to arrive at a definite diagnosis.

\section{Analysis of cases \\ Cases were classified into the following six groups.}

Group A: patients with clear-cut evidence of PIND and sufficient data to make a definite diagnosis as to etiology. This group was then further classified by specific diagnosis.

Group B: patients with a history compatible with the diagnosis of either the classic or variant forms of CJD. Diagnostic clinical criteria for inclusion in the classic form of this disease included a progressive dementia, typical EEG pattern, and at least two of the following features: myoclonus, visual/cerebellar ataxia, pyramidal/extra pyramidal signs, or akinetic mutism. For inclusion in the variant form of CJD, the patient needed a psychiatric presentation consisting of anxiety, depression or other personality changes in association with progressive deterioration in neurological function. This deterioration could include progressive development of cerebellar signs, memory impairment, myoclonus/chorea, and extrapyramidal/pyramidal signs without EEG changes suggestive of classic form of the disease. Any patient felt to meet the criteria for diagnosis of either form of CJD was referred to the CJD-Surveillance System Canada for confirmation of the diagnosis and follow-up.

Group C: patients with clinical evidence of progressive intellectual and neurological deterioration for which the panel of experts was unable to reach a definite clinical diagnosis as to etiology.

Group D: case reports that were discarded because they did not meet the study case definition once detailed questionnaires were completed.

Group E: case reports that could not be classified due to insufficient clinical information even after numerous attempts had been made to obtain the missing information either by phone or mail. The reason for the lack of information was recorded in each case. 


\section{Table 1: Canadian Paediatric Surveillance Program initial return rates by province/territory (as percentage of physicians surveyed)}

\begin{tabular}{lrrrc}
\hline Province/Territory & 1999 & 2000 & 2001 & $\begin{array}{c}\text { Current number } \\
\text { of participants }\end{array}$ \\
Alberta & 88 & 89 & 88 & 227 \\
British Columbia & 81 & 81 & 76 & 245 \\
Manitoba & 86 & 85 & 86 & 117 \\
New Brunswick & 74 & 81 & 84 & 29 \\
Newfoundland & 73 & 78 & 82 & 42 \\
Northwest Territories & & & & \\
$\quad$ \& Nunavut & 100 & 100 & 100 & 3 \\
Nova Scotia & 88 & 89 & 90 & 78 \\
Ontario & 84 & 82 & 82 & 913 \\
Prince Edward Island & 86 & 96 & 91 & 7 \\
Quebec & 81 & 80 & 78 & 628 \\
Saskatchewan & 67 & 68 & 61 & 47 \\
Yukon & 100 & 100 & 100 & 1 \\
Canadian average & 83 & 82 & 81 & \\
Total number of & & & & 2,337 \\
$\quad$ participants & 2,216 & 2,296 & 2,337 & \\
\hline
\end{tabular}

Group $\boldsymbol{F}$ : duplicate reports were identified and only the initial report was counted.

Totals for each group were reported both nationally and regionally. As this was a descriptive study, prevalence and incidence rates were not calculated.

This study had been reviewed and approved by independent ethics committee.

\section{RESUlts}

Over the study duration, the number of paediatricians and paediatric subspecialists enrolled in the CPSP increased from 2,216 in 1999 , to 2,256 in 2000 , and 2,337 in 2001. Initial reporting rates were $83 \%$ in $1999,82 \%$ in 2000 and $81 \%$ in 2001 , with no significant differences in these rates between provinces (Table 1). The response rate for voluntary completion of detailed questionnaires remained constant at an overwhelming $95 \%$ for 1999, 2000 and 2001. These numbers are similar to other countries using a similar form of surveillance., ${ }^{2,3}$

In all, a total of 99 cases were reported on the initial report form as possible cases meeting the PIND study criteria (Table 2). Sixty cases were classified as having a progressive intellectual and neurological disorder. Fifteen cases did not meet the inclusion criteria. Most of these conditions represented a static encephalopathy secondary to birth asphyxia or trauma. Nine cases could not be classified, as the clinical information was incomplete. Most commonly, the reporting physician could not recall the details necessary to complete the detailed questionnaire. Fifteen cases were duplicate reports of the same index case.

Of the 60 confirmed PIND cases, 52 were attributed to many rare neurodegenerative disorders as listed in Table 3. One case of iatrogenic CJD, reported in 1999, involved a 13-year-old child who underwent a duraplasty at three years of age using cadaver dural matter and presented ten years later. In addition to five different reports of this case to the CPSP, the case was also reported independently to the CJD Surveillance System Canada. No cases of vCJD were found during the period of study surveillance. In the other eight PIND cases, a definite etiological diagnosis could not be attained even after exhaustive investigations and review by the expert panel.

\section{Discussion}

The timing and methodology of the CPSPstudy was modeled after the British Paediatric Surveillance Unit study to allow for comparison of the data results. ${ }^{4}$ Each national study did, however, differ slightly as a result of geographic and population differences between the two countries. As a result of the smaller geographic distance in the UK, the British Paediatric Surveillance Unit study was able to have a study nurse visit the reporting physician to collect the needed data whereas, in Canada, the reporting physician completed the questionnaire and was contacted with a follow-up telephone interview if needed. As paediatric neurologists and medical geneticists in Canada are at all major provincial teaching hospitals, diagnoses that had

Table 2: Breakdown of progressive intellectual and neurological deterioration reports by province/territory

\begin{tabular}{|c|c|c|c|c|c|c|}
\hline Provinces & Total & PIND & $\begin{array}{l}\text { Average incidence } \\
\text { per million children }\end{array}$ & Not PIND & Incomplete & Duplicate \\
\hline British Columbia/Yukon & 6 & 3 & 1.66 & 1 & 1 & 1 \\
\hline Alberta & 12 & 11 & 7.2 & 0 & 0 & 1 \\
\hline Saskatchewan & 2 & 2 & 3.72 & 0 & 0 & 0 \\
\hline Manitoba & 1 & 1 & 1.72 & 0 & 0 & 0 \\
\hline Ontario & 47 & 27 & 4.92 & 10 & 7 & 3 \\
\hline Quebec & 13 & 7 & 2.19 & 2 & 1 & 2 \\
\hline New Brunswick & 5 & 2 & 6.00 & 1 & 0 & 2 \\
\hline Nova Scotia & 5 & 2 & 4.75 & 0 & 0 & 3 \\
\hline Newfoundland & 8 & 5 & 21.8 & 1 & 0 & 2 \\
\hline TOTALS & 99 & 60 & 4.21 & 15 & 9 & 15 \\
\hline
\end{tabular}


Table 3: Etiological diagnoses of progressive intellectual and neurological deterioration cases

\begin{tabular}{lcc}
\hline Etiology & Number of cases & $\begin{array}{c}\text { Average incidence } \\
\text { per million children }\end{array}$ \\
Creutzfeldt-Jakob disorder & 1 & 0.7 \\
Adrenoleukodystrophy & 2 & 0.14 \\
Alexander's disorder & 1 & 0.07 \\
Ceriod lipofuscinosis & 8 & 0.56 \\
Mucopolysaccharidosis & 7 & 0.49 \\
Glucose transporter defect & 1 & 0.07 \\
Krabbe's disorder & 5 & 0.35 \\
Lafora disorder & 1 & 0.07 \\
Mitochondrial disorders & 16 & 1.12 \\
Neuman-Pick type C disorder & 2 & 0.14 \\
Rett's disorder & 4 & 0.28 \\
Vanishing White Matter disorder & 4 & 0.28 \\
Undetermined etiology & 8 & 0.56 \\
\hline
\end{tabular}

been previously confirmed by either a Canadian paediatric neurologist or medical geneticist were accepted without review by the expert panel. In contrast, a panel of experts reviewed all cases in the British study.

The results reported in this paper are for the most part similar to those reported by the British study, ${ }^{3}$ as the many different etiologies and relative reporting frequencies of progressive intellectual and neurological disorders were similar. When our calculated incidences for three most common conditions in our series (mitochondrial disorders, Krabbe's and neuronal lipofuscinoses) were compared to those reported in GeneReviews, ${ }^{5}$ no significant differences were found. This would suggest that the results of this surveillance program were fairly accurate. As well, both programs had a similar number of PIND cases for which definite etiological diagnoses could not be made. One significant difference between the British and Canadian study results was in the number of cases of Tay-Sachs disease. This gangliosidosis diagnosis accounted for a significant number (59) of the British cases identified by the British Paediatric Surveillance Unit, whereas, none were identified in Canada. This difference might be a reflection of the current active genetic-screening program targeting at-risk populations in Canada.

In contrast to the six cases of vCJD identified in the United Kingdom, none were reported in Canada. ${ }^{4}$ As both surveillance studies were ongoing during roughly the same time period, this finding strengthens the epidemiological evidence that the exposure to the causative agent was most probably unique to the United Kingdom.

The CJD case was correctly diagnosed and reported to both the CPSP and the CJD Surveillance System Canada. But could other cases have been misdiagnosed? A careful review of all
CPSP case reports meeting the PIND study criteria without having a specific etiological diagnosis confirmed that no cases of either form of CJD were missed. This provided additional validation that CJD Surveillance System Canada ascertainment of both forms of CJD in the paediatric population was complete.

On the one hand, modern methods of diagnosis, treatment and prevention have resulted in a significant decline of conditions previously associated with either high mortality or prolonged or severe morbidity in children living in industrial countries. On the other hand, the contribution of rare diseases to mortality and morbidity becomes even more significant. It is expensive and nearly impossible for an individual investigator to identify a sufficient number of cases to derive meaningful epidemiological data. Yet relying on data collected from Centers of Excellence in the management of a particular disorder can lead to selective and atypical epidemiological data. Soliciting cases by letter writing, advertising or using national disease specific registers established by volunteer lay organizations also has a tendency towards errors in correct diagnosis and bias of ascertainment as not all persons affected with a particular disorder might wish to take part in the registry. Using morbidity and mortality records are complicated by accuracy of diagnostic coding and the fact that some rare disorders do not have a specific International Classification Diagnostic code. The active surveillance method used in this CPSP study is an attempt to circumvent these problems. Relatively speaking, the CPSP is an inexpensive epidemiological surveillance tool. ${ }^{3}$ Accuracy of ascertainment is ensured by the use of a uniform case definition, a selected group of physicians most likely to see children with the disorder under study, an established surveillance system with a high reporting rate, and an expert panel to review diagnoses. As in all voluntary reporting systems relying on busy treating physicians, there is always a certain amount of under-reporting. For reasons of patient confidentiality, the data collected on the initial reporting form were kept to the bare essentials (i.e., date of birth and sex). Cases were lost as result of the treating physician not being able to recall the specific information about a case several weeks after the initial report was completed.

The ethics of establishing patient database for surveillance remains primordial. A balance between the need to maintain patient confidentiality and the need to collect surveillance data of rare conditions is necessary. In the United Kingdom, the Data Protection Act (1998) requires that consent be obtained before identifiable data necessary for public health surveillance can be shared between health professionals and surveillance units. ${ }^{6}$ No patient specific information, such as name, hospital number or date of birth can be used. If, in the future, doctors taking part in surveillance programs have to obtain explicit patient or parent consent to share data with other health professionals, response rates could decrease because of parental refusal and increased physician workload, as shown by the British experience. ${ }^{6}$ The timelines of data collection and analysis could be significantly increased as well as a result of delays in obtaining consent.

In conclusion, this CPSP PIND study illustrated that paediatricians treating children with PIND are facing a challenging task as a myriad of neurodegenerative disorders can present in a similar fashion and that even after exhaustive investigations and expert panel review, some children will never attain a diagnosis. The study confirmed that no new cases of 
vCJD were discovered in Canada and provided validation that surveillance for CJD is working. The possibility of using similar methods of active surveillance can provide useful public health information about trends occurring in rare neurological disorders within and between countries. It also opens the door to collaboration and cooperation between researchers in different national surveillance units that are members of the International Network of Paediatric Surveillance Units.

\section{ACKNOWLEDGEMENTS}

The Division of Blood-borne Pathogens Laboratory, Centre for Infectious Disease Prevention and Control, Health Canada funded this study. We thank Ms. Andrea Medaglia, Senior Coordinator for the Canadian Paediatric Surveillance Program for managing the overall administrative details of the study; Drs. Peter Humphreys, Daune MacGregor, Frederick Andermann, Annette Feigenbaum, and Jean Michaud for being part of the panel of experts who reviewed all the cases; and the CPSP participants who completed the questionnaires as part of the surveillance program.

\section{REFERENCES}

1. Sockett PN. Canadian paediatric surveillance program: two years of a system for investigating unusual paediatric disorders. Paediatr Child Health 1998 3:240-245.

2. Nicoll A, Lynn R, Rahi J, Verity C, Haines L. Public health outputs from the British Paediatric Surveillance Unit and similar clinicalbased systems. J R Soc Med 2000 93:580-585.

3. Hall SM, Nicoll A. The British pediatric surveillance unit-a pioneering method for investigating the less common disorders of childhood. Report of a seminar held in June 1995. Child Care Health Dev 1998 24:129-143.

4. Verity CM, Nicholl RG, Devereux G, Stellitano L. Variant Creutzfeldt-Jakob disease in UK children: a national surveillance study. Lancet 2000 356:1224-1227.

5. GENE REVIEWS University of Washington, Seattle, www.genetest.org

6. Verity CM, Nicoll A. Consent, confidentiality, and the threat to public health surveillance. Br Med J 2002; 324: 1210-1213. 